\section{Gategory}

Synthesis of

Materials and

Unnatural Products

\section{Key words}

fluorophores

Stille coupling

dyes
H. WANG, F. R. FRONCZEK, M. G. H. VinCENTE, K. M. SMITH* (LOUISiAnA STATE UNIVERSITY, BATON ROUGE, USA)

Functionalization of 3,5,8-Trichlorinated BODIPY Dyes

J. Org. Chem. 2014, 79, 10342-10352.

\title{
Phosgene as Key Reagent for Trifunctionalization of BODIPY Dyes
}<smiles>Cc1[nH]c(C(=O)O)c(C)c1[18O]</smiles>

1. $\mathrm{Br}_{2}$ (1 equiv)

$\mathrm{Et}_{2} \mathrm{O}$, r.t., overnight

2. $\mathrm{MeOH}$, reflux, overnight

$70 \%$ yield

$\mathrm{BnO}_{2} \mathrm{C}$

$\mathrm{R}^{1}=\left(\mathrm{CH}_{2}\right)_{2} \mathrm{CO}_{2} \mathrm{Me}$<smiles>Cc1c(Cl)c2n(c1Cl)B(F)P(F)n1c(I)c(I)c(Cl)c1-2</smiles>

$\mathrm{Pd}\left(\mathrm{PPh}_{3}\right)_{4}(0.05$ equiv) $\mathrm{PhMe}$, reflux, $5 \mathrm{~h}$

1. DIPEA (7 equiv) $\mathrm{CHCl}_{3}$, r.t., $30 \mathrm{~min}$

2. $\mathrm{BF}_{3} \cdot \mathrm{OEt}_{2}$ (10 equiv) r.t., $24 \mathrm{~h}$

$55 \%$ yield<smiles>Cc1ccccc1Cc1[nH]c(C(=O)OCc2ccccc2)c(C)c1C(=O)OCc1ccccc1</smiles>

1. $\mathrm{Pb}(\mathrm{OAc})_{4}$ (2.2 equiv) $\mathrm{AcOH}$, r.t., $4 \mathrm{~d}$

2. $\mathrm{PbO}_{2}$ (2.9 equiv), $2 \mathrm{~d}$

$60 \%$ yield

1. $\mathrm{Pd} / \mathrm{C}, \mathrm{H}_{2}$ THF, r.t., overnight

2. $I_{2}$ ( 1.5 equiv)

$\mathrm{NaHCO}_{3}$ (5.1 equiv) $\mathrm{H}_{2} \mathrm{O}-\mathrm{MeOH}$, r.t., $2 \mathrm{~h}$,
Significance: The functionalization of BODIPY fluorescent dyes allows the tuning of their photophysical, hydrophobic, and charge-transfer properties. Smith and co-workers utilize phosgene to access a 3,5,8-trichlorinated BODIPY. Exploiting the higher reactivity of the vinylic chloride in Stille couplings, regioselectively functionalized BODIPY dyes are synthesized.
Comment: Starting from a trichlorinated BODIPY and performing mixed Stille couplings with only four different organotin reagents, 14 new BODIPY dyes are reported in this paper. Photophysical properties are tunable via substitution at the 3-, 5-, and 8-positions. A plethora of regioselectively functionalized BODIPY dyes should be accessible through this strategy.

SYNFACTS Contributors: Timothy M. Swager, Sibo Lin

Synfacts 2015, 11(1), 0034 Published online: 15.12.2014 Dol: 10.1055/s-0034-1379663; Reg-No.: S12214SF 